\title{
Recurrent renal cell carcinoma manifesting as a large intrathoracic fibrotic mass: A case report
}

\author{
JI HYUN KIM, JAE HOON JEONG, SUNG-HYUN PARK, JIN SEON JEONG, \\ YOUNG-JOON RYU and SEO-YOUNG SONG
}

\begin{abstract}
Department of Internal Medicine, Kangwon National University Hospital, Kangwon National University, School of Medicine, Chuncheon, Gangwon 200-947, Republic of Korea
\end{abstract}

Received March 18, 2015; Accepted April 4, 2016

DOI: $10.3892 / 01.2016 .4460$

\begin{abstract}
Renal cell carcinomas (RCCs) have a strong tendency to metastasize, and the most common sites are the lungs, bones and liver. Late recurrence is another feature of the RCC, with lesions appearing $\geq 10$ years after surgical treatment. However, fibrosis has rarely been associated with the disease. The present study reports a case of recurrent RCC that manifested as a fibrotic mass within the thorax. A 48-year-old man presented with dyspnea that had persisted for 3 days. The patient had undergone a right radical nephrectomy for stage II clear cell carcinoma of the kidney 6 years previously. The patient was a current smoker, with a smoking history of 20 pack-years. Chest radiography showed pleural effusion in the right thorax with an egg-sized mass shadow within the right upper lung (RUL) field. Computed tomography (CT) showed a main mass, $7 \mathrm{~cm}$ in diameter, within the RUL, with heterogeneous enhancement and multiple nodules of various sizes in the lungs, suggestive of primary lung cancer or metastatic RCC. A CT-guided percutaneous needle aspiration biopsy was obtained from the main mass, but histology revealed dense fibrous tissue without any malignant cells. Positron emission tomography-CT demonstrated an irregular hypermetabolic RUL mass, with a standardized uptake value (SUV) of 5.0, along the right pleura, and small pulmonary nodules (SUV, 2.0). Ultrasound-guided biopsy was attempted for a smaller hypermetabolic pleural nodule and the result was clear cell adenocarcinoma, consistent with the previous renal histology. The present study describes the case, along with a review of the relevant literature.
\end{abstract}

Correspondence to: Dr Seo-Young Song, Department of Internal Medicine, Kangwon National University Hospital, Kangwon National University, School of Medicine, 17-1 Hyoja 3-Dong, Chuncheon, Gangwon 200-947, Republic of Korea

E-mail: sysong@kangwon.ac.kr

Key words: renal cell carcinoma, recurrent, intrathoracic, metastasis, fibrosis

\section{Introduction}

Renal cell carcinomas (RCCs) originate from the renal cortex and constitute $90 \%$ of all primary renal neoplasms (1). RCCs have a strong tendency to metastasize, most commonly to the lungs, bones and liver (2). However, RCC is also renowned for unpredictable patterns of secondary spread involving almost every other site in the body due to the complex lymphatic drainage of the kidneys (3). According to National Comprehensive Cancer Network guideline, localized RCC may be effectively treated with surgery alone, while some patients with metastatic RCC may also benefit from it (3). Patients who develop solitary recurrence within the lung, bone or brain may be candidates for surgical resection of primary and metastatic tumor. Systemic therapy for metastatic RCC can be differentiated as for cell type; first-line therapy for patients with predominantly clear cell type include targeted therapy with sunitinib, sorafenib, pazopanib, axitinib, temsirolimus, everolimus, bevacizumab with interferon; treatments for non-clear cell carcinoma include temsirolimus, everolimus, sunitinib, sorafenib, pazopanib, axitinib, or erlotinib (3). Late recurrence is another feature of RCC, with metastatic lesions appearing $\geq 10$ years after surgical treatment; however, fibrosis has rarely been associated with RCC (3). A few studies have appeared describing retroperitoneal and perirenal fibrosis associated with RCC (4-6). The current study presents a case in which the first metastatic recurrence of RCC manifested as a fibrotic mass in the thoracic cavity 6 years after a radical nephrectomy. Written informed consent was obtained from the patient for publication of this case study.

\section{Case report}

A 48-year-old man with dyspnea that had persisted for 3 days visited the emergency department of Kangwon National University Hospital of Chuncheon (Chuncheon, Korea) in March, 2014. The patient was a current smoker, with a smoking history of 20 pack-years. The patient had undergone a right radical nephrectomy for stage II clear cell carcinoma of the kidney 6 years previously. At this time, the chest radiography was unremarkable (Fig. 1A), and the patient received regular follow-ups with no signs of metastatic disease. Upon the current admission, chest radiography revealed pleural effusion 

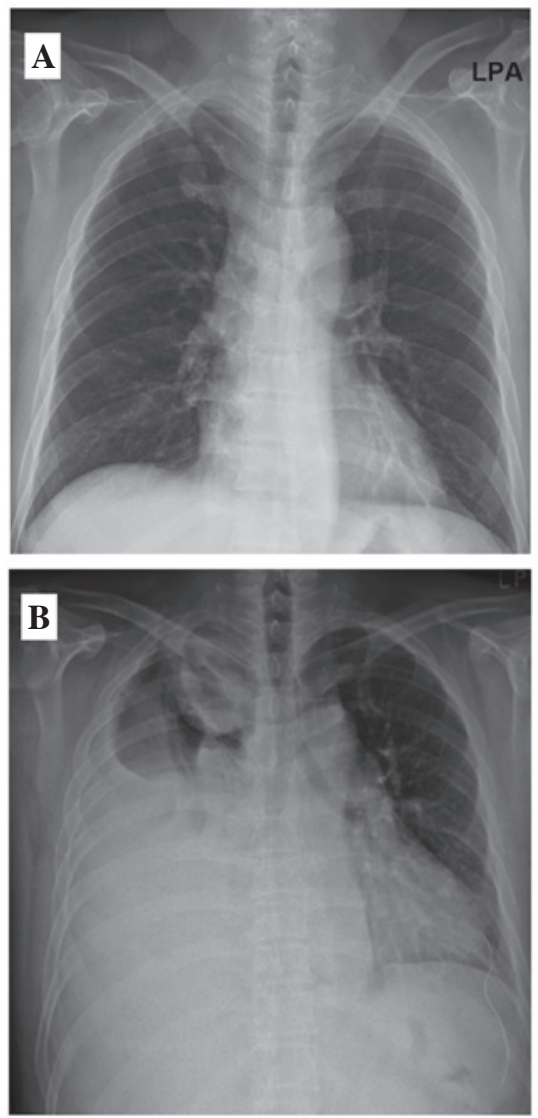

Figure 1. Chest radiography. (A) Initial chest radiography was normal, (B) but 6 years later, pleural effusion with an egg-sized mass on the right upper lung field was observed. LPA, left side of the patient, posterior-anterior view
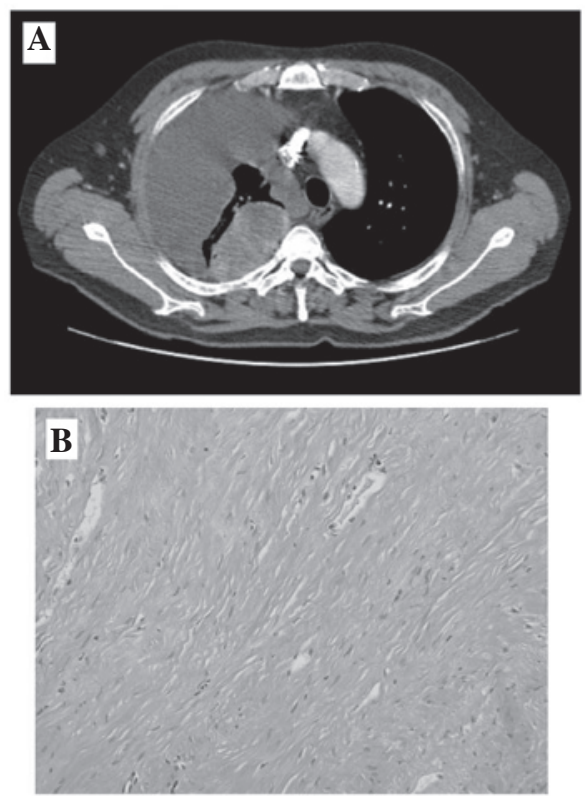

Figure 2. Chest CT scan and histology of RUL mass. (A) CT showing a round mass, $7 \mathrm{~cm}$ in diameter, with heterogeneous enhancement. (B) Microscopic appearance of tissue from the RUL mass showing fibrosis without malignant cells (hematoxylin and eosin; magnification, x100). CT, computed tomography; RUL, right upper lung.

in the right thorax with an egg-sized mass shadow within the right upper lung (RUL) field (Fig. 1B). Upon examination,
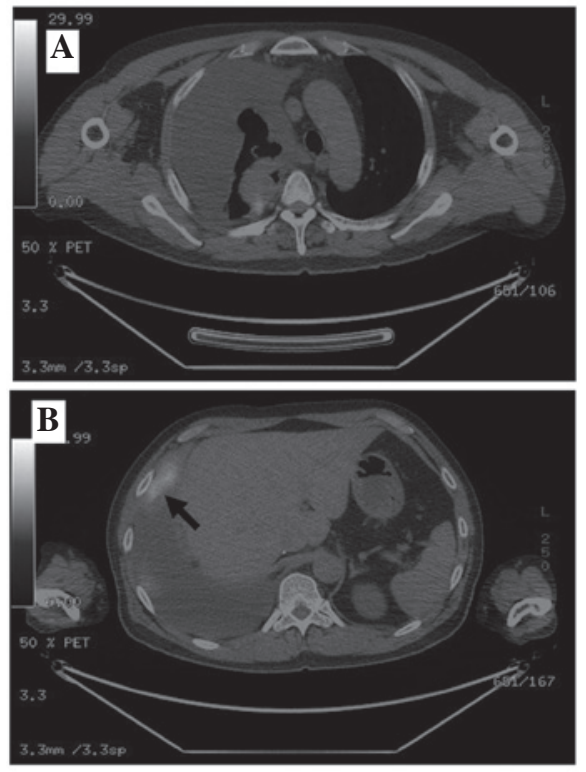

Figure 3. Fluorodeoxyglucose positron emission tomography-computed tomography. (A) Irregular hypermetabolic mass with nodular thickening, at the previous biopsy site. (B) Hypermetabolic mass on the right lower lung field, at the second biopsy site (arrow).
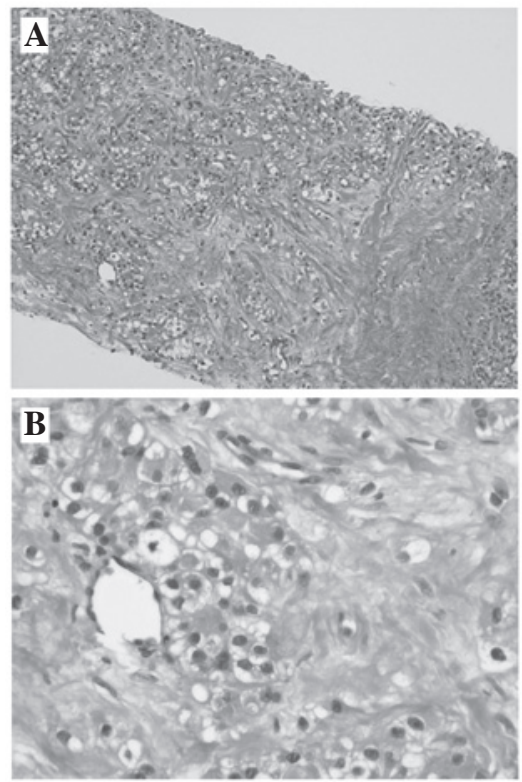

Figure 4. Histological features from the pleural nodule. (A) Microscopic appearance showing infiltration of tumor cells (H\&E; magnification, x100). (B) High-powered view showing cancer cells with clear cell type, consistent with metastatic clear cell renal cell carcinoma (H\&E; magnification, $\mathrm{x} 400)$. $\mathrm{H} \& \mathrm{E}$, hematoxylin and eosin.

the patient's vital signs were within normal limits, but breath sounds were diminished for the right lung. Laboratory data showed the following: White blood cell count, $10,100 / \mathrm{mm}^{3}$

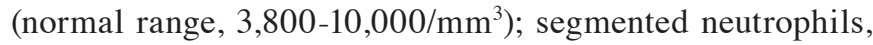
$70 \%$ (normal range, $40-70 \%$ ); lymphocytes, $18 \%$ (normal range, 20-50\%); monocytes, $8 \%$ (normal range, 3-9\%); eosinophils, $2 \%$ (normal range, $0-5 \%$ ); hemoglobin, $13.2 \mathrm{~g} / \mathrm{dl}$ (normal range, 13.3-16.5 g/dl); serum lactate dehydrogenase (LDH), $336 \mathrm{U} / 1$ (normal range, <190 U/l); and total protein, $8.2 \mathrm{~g} / \mathrm{dl}$ 
(normal range, 5.7-8.2 g/dL). Thoracentesis was performed, and the pleural fluid was serosanguinous in color, with a $\mathrm{pH}$ of 7.0 . The red blood cell count was $134,000 / \mathrm{mm}^{3}$, the white blood cell count was $410 / \mathrm{mm}^{3}$ (polymorphonuclear cells, 14\%; and mononuclear cells, $80 \%$ ), the glucose level was $132 \mathrm{mg} / \mathrm{dl}$, the protein level was $0.626 \mathrm{~g} / \mathrm{dl}$ and the LDH level was $523 \mathrm{U} / 1$. As the patient had been diagnosed with $\mathrm{RCC}$ in the past, and the results of examination had shown unilateral monocyte-dominant exudative pleural effusion with a mass shadow on X-ray, a computed tomography (CT) scan was now performed. CT showed a round mass, $7 \mathrm{~cm}$ in diameter, on the RUL, with heterogeneous enhancement, and multiple nodules of various sizes in the lungs, suggestive of primary lung cancer or metastatic RCC (Fig. 2A). To determine the correct differential diagnosis between primary cancer of the thorax, such as lung cancer or mesothelioma, and metastatic recurrence of RCC, a CT-guided percutaneous needle aspiration biopsy was performed on the main mass. However, histopathological assessment revealed a dense fibrous lesion without malignant cells (Fig. 2B). Briefly, biopsy tissues were fixed by $10 \%$ formaldehyde (overnight), then paraffin embedded (PE) blocks were produced. They were cut at $4-\mu \mathrm{m}$ thickness, mounted onto slides, and then stained using a hematoxylin and eosin stain (Dako, Glostrup, Denmark) protocol. For immunohistochemical staining, slides were incubated overnight at $4{ }^{\circ} \mathrm{C}$ with primary antibodies, including monoclonal mouse Wilms tumor protein (cat. no. 6F-H2; ready to use; Cell Marque ${ }^{\mathrm{TM}}$; Sigma-Aldrich, St. Louis, MO, USA), monoclonal rabbit anti-mouse calretinin (cat. no. RM-9113-S0; 1:100; Thermo Fisher Scientific, Inc., Waltham, MA, USA), monoclonal mouse anti-human cytokeratin 5/6 (cat. no. M7237; 1:50; Dako) and monoclonal mouse anti-human thyroid transcription factor-1 (cat. no. PA0364; ready to use; Bond ${ }^{\mathrm{TM}}$; Leica Biosystems, Wetzlar, Germany). Immunohistochemical staining was performed using an auto stainer (XT System Benchmark, Ventana Medical System, Tucson, AZ, USA) according to the manufacturer's instruction. The pleural fluid was sent for cytological examination twice, and demonstrated no evidence of malignancy. However, due to a strong clinical suspicion of malignancy, positron emission tomography-CT (PET-CT) was performed, which demonstrated an irregular hypermetabolic RUL mass, with nodular thickening along the right pleura, with a standardized uptake value (SUV) of 5.0, and small pulmonary nodules with an SUV of 2.0 (Fig. 3A and B). Ultrasound-guided biopsy of a hypermetabolic pleural nodule visualized on PET-CT was attempted, and histological assessment found the lesion to consist of tumor cells with abundant clear cytoplasm and small round nuclei with atypia, consistent with a diagnosis of metastatic clear cell carcinoma (Fig. 4). Immunohistochemical analyses were negative for Wilms tumor protein, calretinin, cytokeratin 5/6 and thyroid transcription factor-1, which excluded mesothelioma or primary lung cancer from the final diagnosis. The patient was diagnosed with recurrent RCC and treatment was commenced with $50 \mathrm{mg}$ oral sunitinib daily. Although there was a significant decrease in the amount of pleural effusion after 1 month of treatment with sunitinib, the fibrotic mass did not respond to this medication. Everolimus was administered 2 months later, as the patient developed progressive disease. At present, the patient has exhibited stable disease for 9 months with everolimus treatment.

\section{Discussion}

Renal cell carcinoma accounts for $90-95 \%$ of malignant neoplasms originating from the kidney. The tumors have been reported to be resistant to cytotoxic agents, infrequently responsive to biological response modifiers, such as interleukin-2, and to produce a variable clinical course for patients with metastatic disease (7). Fibrosis is not a usual finding associated with metastatic RCC, and only a few studies have reported retroperitoneal or perirenal fibrosis (4-6).

A previous case report detailed a 53-year-old male who underwent radical nephrectomy for sarcomatoid renal cell carcinoma, and a follow up CT 6 months after surgery demonstrated left retroperitoneal mass which was histologically revealed as a retroperitoneal fibrosis rather than a relapse (4). Another case reports a 78-year-old male diagnosed with clear cell carcinoma where biopsy of the resected mass revealed stromal fibrosis (5), and there was a case of a 43-year-old male with clear cell RCC, which was accompanied by fibrosis of the peritoneum during the surgery (6).

It has been widely accepted that fibrosis is an inflammatory immunological reaction, and although diverse diseases and molecular pathways initiate the fibrotic process, in all instances the biochemical and cellular mechanisms contributing to the final outcome are shared. Tissue injuries activate the immune system and repair mechanisms, which lead to effective healing pathways if a type $1 \mathrm{~T}$ helper (Th1) cell response is dominant, whereas if a Th 2 response is predominant, an increase in Th17 cells will lead to chronic inflammation, ultimately resulting in fibrosis (8). The chronic inflammatory microenvironment has long been recognized as conducive to tumorigenesis. Mesodermal tumor cells produce extracellular matrix molecules involved in fibrosis, whereas ectoderm- or endoderm-derived tumors can undergo epithelial-mesenchymal transition (EMT) under inflammatory conditions, and EMT of parenchymal tumor cells such as in RCC, is crucial for tumor-associated fibrosis and metastasis (9).

Fromowitz and Miller (10) suggested fibrosis as an exaggerated host response to tumor-associated antigens in RCC. The pathogenesis of fibrosis associated with metastatic RCC is uncertain, although a study by Terada (6) suggested that local tissue responses to RCC may protect the surrounding tissue from RCC invasion and growth, and thus fibrosis is considered a favorable reaction for the patient.

The present case is of note since the biopsy of the main mass on the RUL revealed fibrotic tissue, which made it difficult to distinguish between primary lung cancer and metastatic RCC. Few cases have reported on retroperitoneal and perirenal fibrosis associated with RCC (4-6). However, to the best of our knowledge, no cases of fibrosis associated with pleural or lung metastasis of RCC have been reported. It was also a possibility that the main mass could have been a benign fibrotic mass in the present study, however, the PET-CT scan showed a lesion with focal fluorodeoxyglucose uptake in the nodular mass, surrounded by fibrotic tissue, suggesting malignancy and a fibrotic response. The role of PET-CT for RCC has been limited to restaging or the evaluation of 
metastasis (11-13). However, PET-CT may have a complementary role as a problem-solving tool in cases that are equivocal on conventional imaging.

In summary, the present report describes a patient in whom recurrent RCC manifested in the thorax as lung and pleural metastases, including fibrosis. It is important for physicians to rule out primary lung cancer or mesothelioma for the differential diagnosis through use of multimodalities such as immunohistochemistry and PET-CT.

\section{Acknowledgements}

This study was supported by a grant from the Kangwon National University (grant no. 20141482).

\section{References}

1. Chow WH, Dong LM and Devesa SS: Epidemiology and risk factors for kidney cancer. Nat Rev Urol 7: 245-257, 2010.

2. Gupta K, Miller JD, Li JZ, Russell MW and Charbonneau C: Epidemiologic and socioeconomic burden of metastatic renal cell carcinoma (mRCC): A literature review. Cancer Treat Rev 34: 193-205, 2008.

3. Sountoulides P, Metaxa L and Cindolo L: Atypical presentations and rare metastatic sites of renal cell carcinoma: A review of case reports. J Med Case Rep 5: 429, 2011.

4. Esquena S, Abascal JM, Trilla E, Torres I and Morote J: Case report: Retroperitoneal fibrosis simulating local relapse of sarcomatoid renal cell carcinoma. Int Urol Nephrol 38: 463-465, 2006.
5. Kumar Y, Bhatia A, Das A and Kathpalia AS: Unusual appearance of perirenal fibrosis in renal cell carcinoma simulating a tumour. Jpn J Clin Oncol 39: 677-681, 2009.

6. Terada T: Retroperitoneal fibrosis associated with renal cell carcinoma. Int J Clin Exp Pathol 6: 1195-1196, 2013.

7. Cohen HT and McGovern FJ: Renal cell carcinoma. N Engl J Med 353: 2477-2490, 2005.

8. Wick G, Grundtman C, Mayerl C, Wimpissinger TF, Feichtinger J, Zelger B, Sgonc R and Wolfram D: The immunology of fibrosis. Annu Rev Immunol 31: 107-135, 2013.

9. Aggarwal BB, Shishodia S, Sandur SK, Pandey MK and Sethi G: Inflammation and cancer: How hot is the link? Biochem Pharmacol 72: 1605-1621, 2006.

10. Fromowitz FB and Miller F: Retroperitoneal fibrosis as host response to papillary renal cell carcinoma. Urology 38: 259-263, 1991.

11. Ramdave S, Thomas GW, Berlangieri SU, Bolton DM, Davis I, Danguy HT, Macgregor D and Scott AM: Clinical role of F-18 fluorodeoxyglucose positron emission tomography for detection and management of renal cell carcinoma. J Urol 166: 825-830, 2001.

12. Safaei A, Figlin R, Hoh CK, Silverman DH, Seltzer M, Phelps ME and Czernin J: The usefulness of F-18 deoxyglucose whole-body positron emission tomography (PET) for re-staging of renal cell cancer. Clin Nephrol 57: 56-62, 2002.

13. Kang DE, White RL Jr, Zuger JH, Sasser HC and Teigland CM: Clinical use of fluorodexoyglucose F 18 positron emission tomography for detection of renal cell carcinoma. J Urol 171: 1806-1809, 2004. 